\title{
Topologically frustrated dynamics in an uncharged Tetra-PEG gel
}

\author{
Di Jia ${ }^{1,2 *}$, Yui Tsuji ${ }^{1,3}$, Mitsuhiro Shibayama ${ }^{3}$, Murugappan Muthukumar $^{1 *}$,
}

1. Department of Polymer Science and Engineering, University of Massachusetts, Amherst, Amherst, Massachusetts 01003, United States

2. Beijing National Laboratory for Molecular Sciences, State Key Laboratory of Polymer Physics and Chemistry, Institute of Chemistry Chinese Academy of Sciences, Beijing 100190, China; University of Chinese Academy of Sciences, Beijing 100049, China

3. Institute for Solid State Physics, The University of Tokyo, Kashiwanoha, Kashiwa 277-8581, Japan

4. Neutron Science and Technology Center, Comprehensive Research Organization for Science and Society, Tokai, Ibaraki 319-1106, Japan

*Corresponding authors: D. Jia: jiadi11@iccas.ac.cn M. Muthukumar: muthu@polysci.umass.edu

\begin{abstract}
Based on our previous discovery of non-diffusive topologically frustrated dynamics in a charged system where electrostatic interactions between the charged guest and the charged host gel play a role (Nature Communications, 2018, 9, 2248; Physical Review Letters, 2021, 126, 057802), we have investigated the onset of this effect in an uncharged gel matrix. Using sodium (polystyrene sulfonate) as the guest macromolecule and the ideal tetra-PEG gel, we find the emergence of the non-diffusive topologically frustrated dynamical state with a hierarchy of segmental dynamics represented by a stretched exponential of exponent $\beta$ around $1 / 3$. Our results demonstrate the universal behavior of the topologically frustrated dynamical state.
\end{abstract}

\section{Introduction}

Movement of very long polymer chains in restricted environments is ubiquitous in separation science and associated technologies, as well as in crowded biological situations [1-23]. While Brownian motion and diffusion of these chains at finite temperature is the axiom of their motion at ambient conditions, a recent experimental discovery [11,12] based on the dynamics of DNA and synthetic polyelectrolytes, trapped inside a hydrogel at room temperature containing essentially water, has revealed that this axiom is wildly deviated at intermediate confinements. The guest polymers are plunged into an extremely long-lived metastable dynamical state, called the topologically frustrates dynamical state. The reason behind this phenomenon has been conjectured to arise from elicitation of numerous entropic traps into which a very long polymer chain is distributed. The hierarchical non-diffusive dynamics observed in the new dynamical state is also conjectured to arise from either polydispersity in the mesh size of the hydrogel or conformational fluctuations of the portions of the chain in various meshes. In view of this, we address in this paper the role of distribution of mesh size of the gel on the onset of the topologically frustrated dynamical state. To reach this goal, we have monitored the dynamics of 
guest poly(styrene sulfonate) molecules trapped inside ideal networks, with narrow mesh size, made from tetra-polyethylene glycol. We report that the topologically frustrated dynamical state still emerges in these ideal gels, showing that the origin of the new phenomenon is due to conformational fluctuations of the various sectors of the guest macromolecule trapped inside the meshes of the host gel.

\section{Experimental section}

\subsection{Materials}

Tetra-amine terminated poly(ethylene glycol) (Tetra-PEG-NH${ }_{2}$ ) with the molecular weight $\mathrm{M}_{\mathrm{w}}=40 \mathrm{kDa}$ and tetra-NHS-glutarate-terminated poly(ethylene glycol) (Tetra-PEG-NHS) with the molecular weight $\mathrm{M}_{\mathrm{w}}=40 \mathrm{kDa}$ were purchased from NOF Co. (Tokyo, Japan). Here NHS represents $\mathrm{N}$-hydroxysuccinimide. Sodium polystyrene sulfonate (NaPSS) with the molecular weight $M_{w}=2270 k D a$ was purchased from Scientific Polymers. Sodium phosphate, disodium phosphate, and sodium chloride were purchased from Sigma-Aldrich. Hydrophilic Polyvinylidene Fluoride (PVDF) filters with the pore size $450 \mathrm{~nm}$ were purchased from Millex Company. Deionized water was obtained from a Milli-Q water purification system (Millipore, Bedford, MA, U.S.A.). The resistivity of deionized water used was $18.2 \mathrm{M} \Omega \mathrm{cm}$.

\subsection{Gel Synthesis and Sample Preparation}

Two types of 4-armed poly(ethylene glycol) (PEG) macromonomers with equal arm length and with different reactive terminal groups were used to synthesize Tetra-PEG gels. Tetra-PEG gels were synthesized by cross-end-coupling of $\mathrm{N}$-succinimidyl (NHS) terminated tetra-PEG macromonomers and amine-terminated tetra-PEG macromonomers following the previous method $^{1-4}$. Tetra-PEG-NH 2 and Tetra-PEG-NHS were dissolved in phosphate buffer to adjust the $\mathrm{pH}$ so that $\mathrm{pH}=7.4$ for Tetra-PEG-NH $\mathrm{N}_{2}$ solution and $\mathrm{pH}=5.8$ for Tetra-PEG-NHS solution. The ionic strength of the buffer solution was tuned by adding $\mathrm{NaCl}$. Phosphate buffer with ionic strength $100 \mathrm{mM}(\mathrm{pH}=6.8)$ was used as the solvent. Equimolar quantities of Tetra-PEG- $\mathrm{NH}_{2}$ and Tetra-PEGNHS were mixed with certain amount of concentrated PSS solutions to reach the targeted concentrations for each species. The polymer concentration of the Tetra-PEG gel was $60 \mathrm{mg} / \mathrm{mL}$ and the NaPSS concentration was $0,5,20$, and $40 \mathrm{mg} / \mathrm{mL}$, respectively. Since light scattering measurement is extremely sensitive to dust, the light scattering tubes were first washed with pure water and acetone separately for several times. After they were dried in the oven overnight, aluminum foil was used to wrap up the tubes and then these tubes were further cleaned by distilled acetone through an acetone fountain setup ${ }^{5,6}$. Then the pre-gel solutions were slowly filtered into a light scattering tube through a $450 \mathrm{~nm}$ PVDF filter to remove the dust. All the sample preparation work was conducted in a super-clean bench to avoid the dust. At least 24 hours were allowed for the completion of the reaction before the light scattering measurements were performed.

\subsection{Static and dynamic light scattering}

SLS and DLS measurements were performed on a commercial spectrometer equipped with a 
multi-t digital time correlator (ALV-5000/E) with Argon laser light source (output power $=400$ $\mathrm{mW}$ ) of wavelength $514.5 \mathrm{~nm}$. DLS measures the intensity-intensity time correlation function $\mathrm{g}_{2}(\mathrm{t})$ by means of a multi-channel digital correlator and related to the normalized electric field correlation function $\mathrm{g}_{1}(\mathrm{t})$ through the Siegert relation ${ }^{7}$. Measurement was conducted at the scattering angle $30^{\circ}, 40^{\circ}, 50^{\circ}, 60^{\circ}$, and $70^{\circ}$, respectively. The relaxation time was obtained by averaging over three different spatial locations within the samples.

\subsection{Data fitting method}

CONTIN method and multiple exponential fitting method were used to analyze the characteristic relaxation rate $\Gamma$ at each angle $e^{7-10}$. Based on $D=\Gamma / q^{2}$, diffusion coefficient $D$ was calculated. Here $q$ is the scattering wavevector $q=(4 \pi n / \lambda) \sin (\theta / 2)$ with the scattering angle $\theta$, wavelength of the incident monochromatic light $\lambda$, and refractive index of the medium $n$. For the correlation functions of NaPSS in the gel matrix, multiple modes were first confirmed by CONTIN method. In the presence of multiple modes and in order to facilitate comparison to a theory, the normalized electric field correlation function $g_{1}(t)$ was fitted by a sum of one or two exponential decays and a stretched exponential function ${ }^{11-13} \cdot \mathrm{g}_{1}(\mathrm{t})$ was fitted using ORIGIN software by minimization of errors between the fitted prediction and the data. Iterations were performed until the best fitting curve was obtained within the tolerance limit. The residuals, which are obtained from the difference between the original data and the fitting curve, are randomly distributed about the mean of zero and do not have systematic fluctuations about their mean.

\section{Results and discussion}

\subsection{Characterization of the gel matrix}

The gel matrix used here was an uncharged Tetra-PEG gel with monodispersed gel mesh size, because the Tetra-PEG gel was made from two types of 4-armed PEG macromonomers with equal arm length crosslinked through their end groups, as shown in Figure 1(e). Static and dynamic light scattering measurements were conducted to characterize the gel matrix. The normalized correlation function $\mathrm{g}_{1}(\mathrm{t})$ and the corresponding relaxation time distribution function obtained from CONTIN fit at the scattering angle $30^{\circ}$ as a typical example is shown in Figure1(a) and 1(b). Typically for gels, the correlation function obtained from DLS shows a dominant decay with several other relaxations due to inherent inhomogeneities from clustering of cross-links and other impurities ${ }^{13-16}$. These inhomogeneities show up as a static electric field, which interferes directly with the scattered electric field from the gel mode. However, here there is only one mode, indicating the gel is very homogenous and clean. And the gel mode is diffusion-like representing the gel elasticity, and the corresponding elastic diffusion coefficient $D_{\text {gel }}$ is $(4.37 \pm 0.15) E-7 \mathrm{~cm}^{2} / \mathrm{s}$ (Figure1c). From static light scattering measurement, the gel mesh size is obtained by using Ornstein-Zernike equation?

$$
\mathrm{I}(\mathrm{q})=\frac{\mathrm{I}(\mathrm{q} \rightarrow 0)}{1+\mathrm{q}^{2} \xi^{2}}
$$


Where $I(q)$ is the static light scattering intensity at the scattering wave vector $q, \xi$ is the correlation length of the gel and here it is used to estimate the gel mesh size. In the plot of $1 / I(q)$ vs $q^{2}$, we get $\xi=(55 \pm 2.4) \mathrm{nm}$ from the square root of the slope/intercept ratio, as shown in Figure $1(\mathrm{~d})$. It is to be noted that the mesh size determined from Ornstein-Zernicke analysis is different from that obtained from DLS, since the latter does not measure the mesh size, but only elastic moduli.
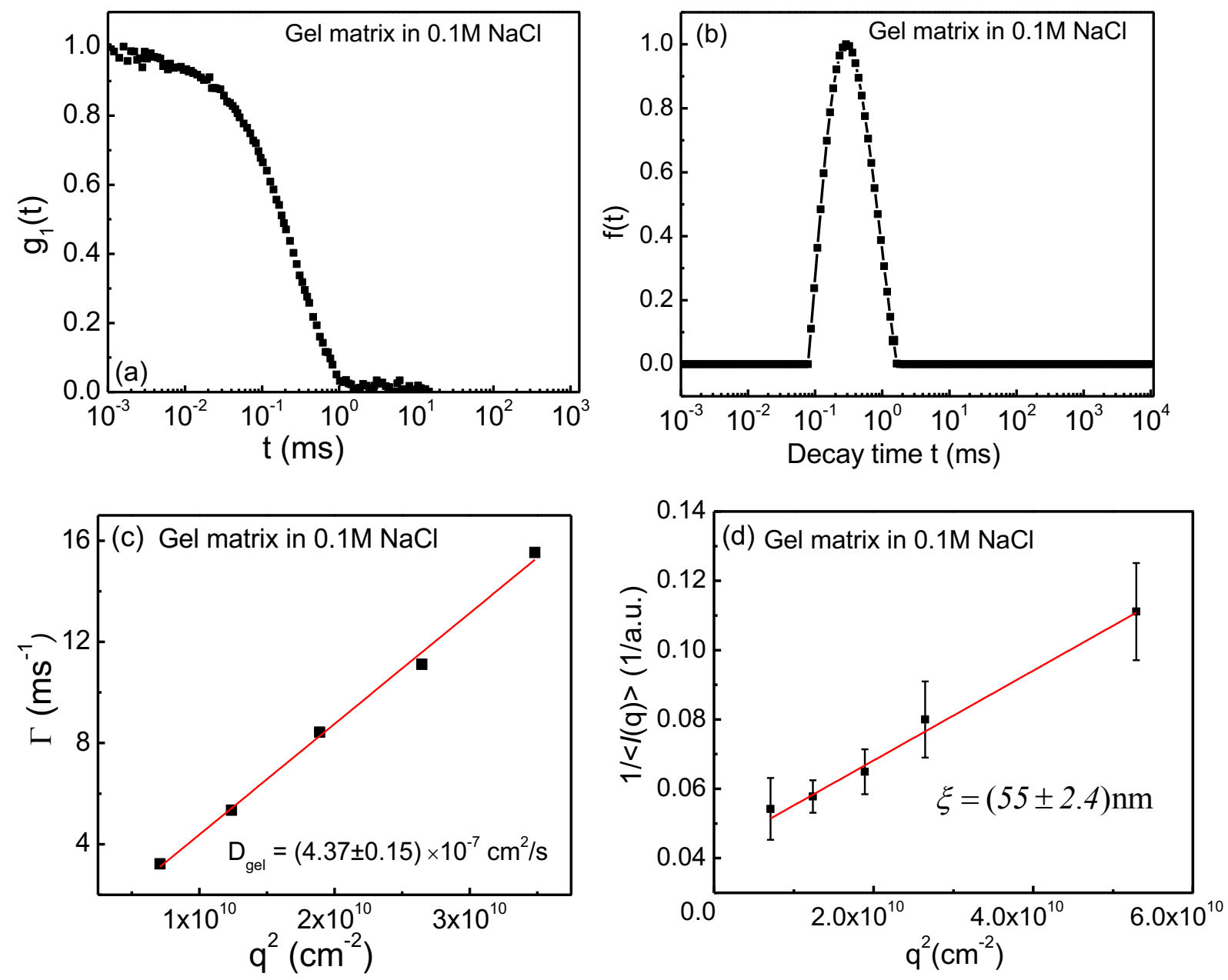

(e)

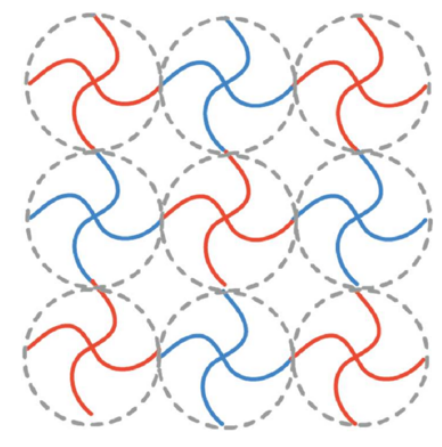

Figure 1. Dynamic and static light scattering results for the Tetra-PEG gel matrix with $0.1 \mathrm{M} \mathrm{NaCl}$. (a) Normalized field-correlation function $\mathrm{g}_{1}(\mathrm{t})$ at scattering angle $30^{\circ}$ measured by dynamic light scattering. (b) Corresponding relaxation time distribution function obtained from CONTIN fit at 
scattering angle $30^{\circ}$. (c) $\mathrm{q}^{2}$ dependence of the relaxation rate $\Gamma$ for the Tetra-PEG gel matrix. (d) Ornstein-Zernike plot of the inverse averaged scattering intensity $1 /<1(q)>$ versus $q^{2}$ measured by static light scattering of the Tetra-PEG gel matrix, yielding the averaged mesh size $\xi=(55 \pm 2.4) \mathrm{nm}$. (e) Schematic cartoon of the Tetra-PEG gel with monodispersed gel mesh size synthesized by crosslinked the end groups of two types of 4-arm macromonomers with equal arm length.

\subsection{Dynamics of guest chains in the Tetra-PEG gel matrix at high concentration}

The guest chain used here is NaPSS with $\mathrm{M}_{\mathrm{w}}=2270 \mathrm{kDa}$ in $0.1 \mathrm{M} \mathrm{NaCl}$ solution. The detailed characterization of NaPSS with $\mathrm{M}_{\mathrm{w}}=2270 \mathrm{KDa}$ in $0.1 \mathrm{M} \mathrm{NaCl}$ solution is in our previous studies ${ }^{10,17}$. For NaPSS with $\mathrm{M}_{w}=2270 \mathrm{KDa}$ in $0.1 \mathrm{M} \mathrm{NaCl}$ solution, the SLS results show that its radius of gyration is $\mathrm{Rg}=96 \mathrm{~nm}$. The DLS results show that there are three diffusive modes representing coupled motion between couterions, co-ions, polyions, and dipole-dipole interaction induced aggregation mode. The key point here is when NaPSS chains are in solutions, all the modes are diffusive. However, it is entirely different when the NaPSS guest chains were imbedded inside the gel matrix.

The best fit of the correlation function $\mathrm{g}_{1}(\mathrm{t})$ for $40 \mathrm{~g} / \mathrm{L}$ NaPSS in the gel matrix can be expressed as (figure 2a):

$$
\mathrm{g}_{1}(\mathrm{t})=\mathrm{a}_{1} \mathrm{e}^{-\Gamma_{1} \mathrm{t}}+\left(1-\mathrm{a}_{1}\right) \mathrm{e}^{-\left(\Gamma_{2} \mathrm{t}\right)^{\beta}}
$$

where $\Gamma_{1}=0.29 \mathrm{~ms}^{-1}$ and $\Gamma_{2}=112.82 \mathrm{~ms}^{-1}$ are the decay rates for the two dynamical modes, $\mathrm{a}_{1}=0.4$ is the weight of the first mode, and the value of the exponent $\beta=0.36$ is a measure of non-diffusive hierarchically dynamical cooperativity of PSS chains in the gel matrix. Analyzing $g_{1}(t)$ data at multiple scattering angles reveals that the first mode (exponential decay) is diffusive, which is demonstrated in Figure $2(\mathrm{~b})$ where $\Gamma_{1} \sim q^{2}$ with the diffusion coefficient $D_{1}=(4.76 \pm 0.08) \mathrm{E}-7 \mathrm{~cm}^{2} / \mathrm{s}$. Since $D_{1}$ is very close to the diffusion coefficient of the gel matrix alone (Figure1c), so we assign the first mode as the gel mode, which indicates the gel elasticity $\left(D_{1}=D_{\text {gel }}\right)$. Then the dynamics of all the guest chains come to the second term in Eq (2), which is a non-diffusive stretched exponential decay, because $\Gamma_{2}$ is not proportional to $q^{2}$ (Figure $2 c$ ). And the value of $\beta$, which indicates the hierarchy of the cooperative local segmental chain dynamics, is independent of scattering angles (Figure $2 \mathrm{~d}$ ), and the averaged value of $\beta$ over all scattering angles is $\beta=$ $(0.33 \pm 0.04)$. The non-diffusive stretched exponential decay of the guest chain dynamics under confinement is ascribed to the non-diffusive topological frustrated dynamics, in which the center of mass of the whole chain cannot diffuse, but within one chain all the segments can do their local segmental dynamics in a hierarchical way. And the $\beta$ values at all scattering angles are within a very narrow range, which is very different from the traditional temperature-dependent glassy and aging systems, where $\beta$ is in the full range $(0<\beta<1)^{18}$. It is also remarkable that all the three diffusive modes in solutions are replaced by a non-diffusive stretched exponential mode when the guest chains are inside the gel matrix.

The discovery here is different from our previous studies, since the previous studies are all for the charged system with a negative charged poly (acrylamide-co-sodium acrylate) gel matrix and either negatively or positively charged polyelectrolytes as the guest chains (Nature 
Communications, 2018 9, 2248; Phys. Rev. Lett. 2021, 126, 057802). While in this work, we have used an uncharged Tetra-PEG neutral gel matrix and NaPSS as the guest chains so that electrostatic interactions between the guest chains and the host gel matrix can be avoided. But still we are able to observe the non-diffusive topologically frustrated dynamics in an uncharged gel matrix, demonstrating such a phenomenon is universal and is ubiquitous for both charged systems and uncharged systems.

Besides, the mesh size distribution of the Tetra-PEG gel matrix is highly monodispersed due to its special synthetic method, while the mesh size distribution of the charged poly(acrylamide-cosodium acrylate) gel is rather polydispersed because it is synthesized by the traditional free radical polymerization ${ }^{19,20}$. And previously we think that the polydispersity of the mesh size in the gel matrix is the possible reason for the emergence of the non-diffusive topologically frustrated dynamics. But here we have observed the non-diffusive topologically frustrated dynamics in a highly monodispersed uncharged gel matrix, demonstrating the emergence of the non-diffusive topologically frustrated dynamics is not due the polydispersity of the mesh size in the gel matrix.
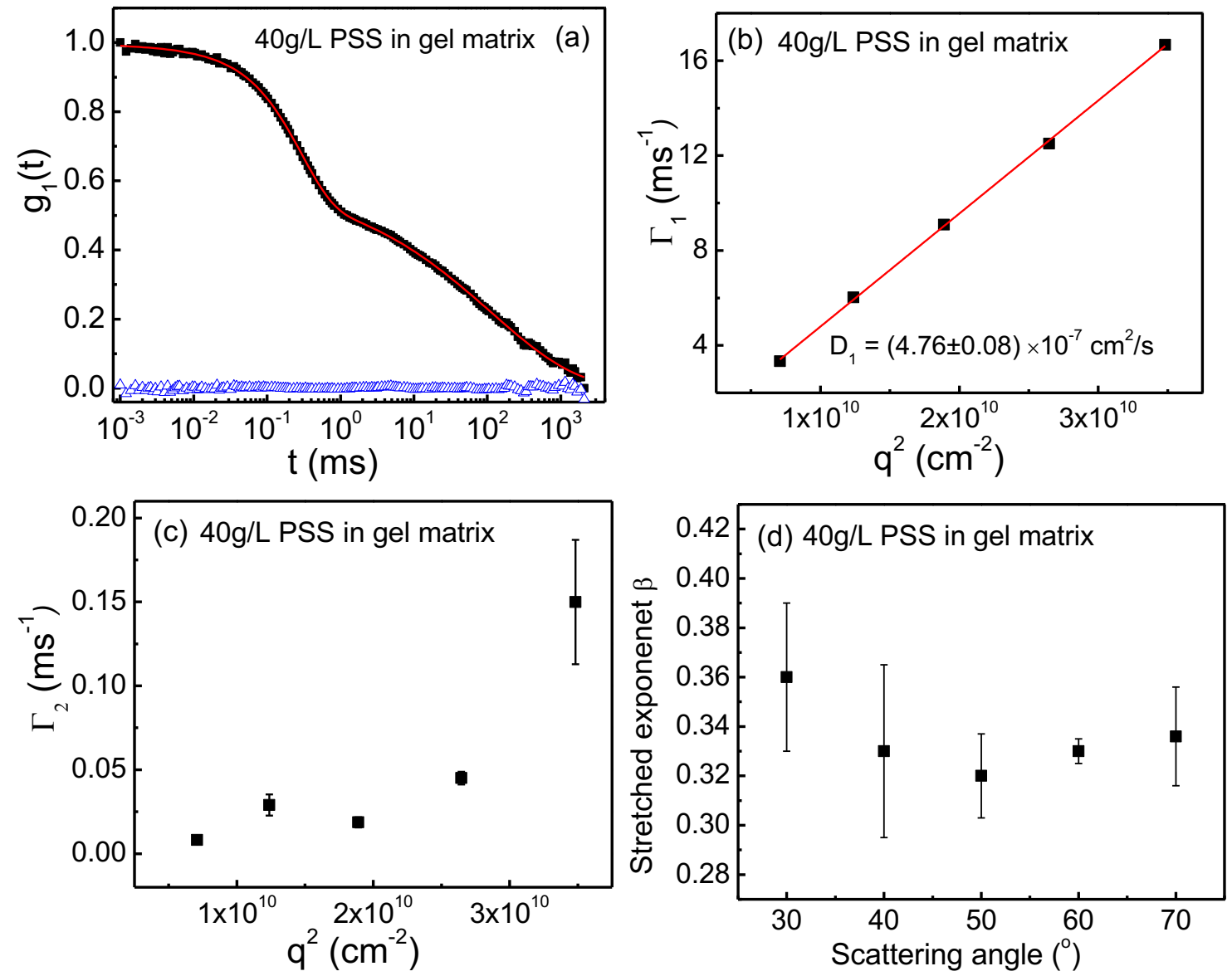

Figure 2. Dynamics of $40 \mathrm{~g} / \mathrm{L}$ NaPSS in the Tetra-PEG gel matrix with $0.1 \mathrm{M} \mathrm{NaCl}$. (a) Normalized field-correlation function $\mathrm{g}_{1}(\mathrm{t})$ at scattering angle $30^{\circ}$. The blue triangles are the residuals between the original data (black) and the fitting curves (red). (b), (c) Fitting results of the relaxation rate $\Gamma$ vs. $q^{2}$ at all angles for the first and second mode, respectively. (d) Stretched exponent $\beta$ as a plot of different scattering angles for $40 \mathrm{~g} / \mathrm{L} \mathrm{NaPSS}$ in the Tetra-PEG gel matrix 
with $0.1 \mathrm{M} \mathrm{NaCl}$.

\subsection{Evidence of weak complexation between NaPSS and the Tetra-PEG gel matrix}

When the concentration of the guest chain in the Tetra-PEG gel is low $(5 \mathrm{~g} / \mathrm{L})$, there are strong fluctuations for the scattered light intensity (Figure 3a) and such strong scattered light intensity fluctuations can easily destroy the scattered light intensity-intensity correlation function $\mathrm{g}_{2}(\mathrm{t})$ so that we cannot obtain the valid correlation function $\mathrm{g}_{2}(\mathrm{t})^{21-23}$. As shown in Figure $3(\mathrm{a})$, for $5 \mathrm{~g} / \mathrm{L}$ NaPSS in the gel matrix, the averaged scattered intensity Is is 30 (a.u.), while the maximum Is is 120 (a.u.), which is 4 times larger than the averaged value. Such strong fluctuations of Is can immediately destroy the scattered light intensity-intensity correlation function $g_{2}(t)$, so that we cannot get the valid $g_{2}(t)$ from the measurement. On the other hand, for high concentration of NaPSS in the gel, such as $40 \mathrm{~g} / \mathrm{L}$ and $20 \mathrm{~g} / \mathrm{L}$, fluctuations in Is becomes smaller by increasing the guest molecules, allowing to obtain a valid scattered light intensity-intensity correlation function $\mathrm{g}_{2}(\mathrm{t})$, as shown in Figure 3(b) and 3(c).
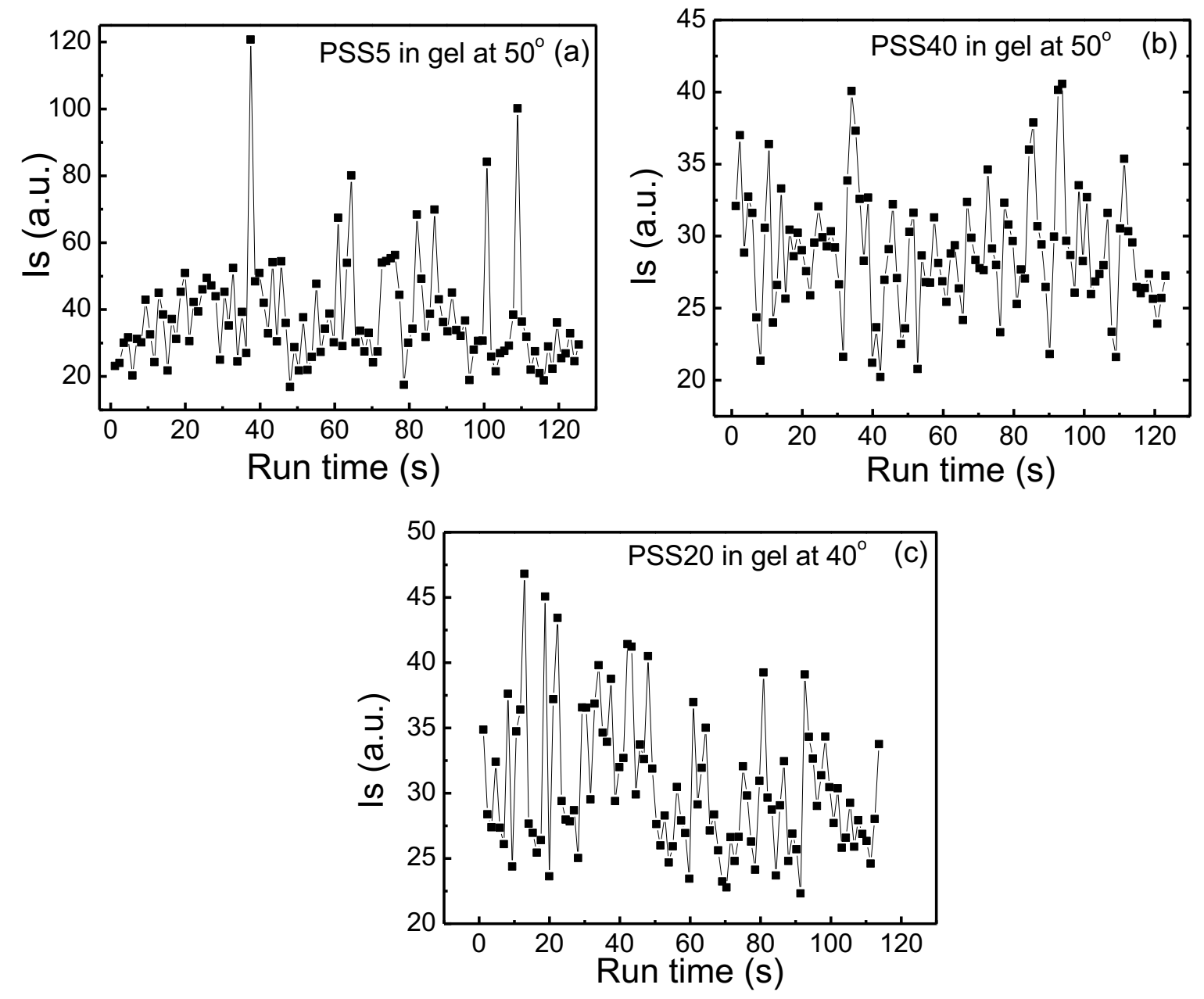

Figure 3. Scattered intensity Is as a function of running time during the measurements for NaPSS of $5 \mathrm{~g} / \mathrm{L}(\mathrm{a}), 20 \mathrm{~g} / \mathrm{L}(\mathrm{b}), 40 \mathrm{~g} / \mathrm{L}(\mathrm{c})$ in the gel matrix, respectively. 
Therefore, we came up with the hypothesis that there is weak complexation between NaPSS chains and the Tetra-PEG gel matrix and such weak complexation can induce strong scattered light intensity fluctuations. When NaPSS concentration is low, all the NaPSS can weakly complex with Tetra-PEG gel because there are enough complexation sites on the gel. However, when NaPSS concentration is high, only part of NaPSS can complex and fully saturated the complexation sites on the Tetra-PEG gel, then the extra free NaPSS chains can move around with no complexation, and the Is signal from the extra free NaPSS chains will dominate over the Is signal from the weakly complexed NaPSS chains. Therefore, we can successfully observe the topologically frustrated dynamics for the extra uncomplexed guest chains.

In order to verify our hypothesis, we have designed an experiment to test the diffusion coefficient $D$ of each species and their mixtures in solutions as follows. We have measured the diffusion coefficients for $5 \mathrm{~g} / \mathrm{L}$ Tetra-PEG- $\mathrm{NH}_{2}\left(\mathrm{M}_{\mathrm{w}}=40 \mathrm{KDa}\right)$ macromonomer solution, $5 \mathrm{~g} / \mathrm{L}$ TetraPEG-NHS macromonomer $\left(\mathrm{M}_{\mathrm{w}}=40 \mathrm{KDa}\right)$ solution, $10 \mathrm{~g} / \mathrm{L} \mathrm{NaPSS}\left(\mathrm{M}_{\mathrm{w}}=68 \mathrm{KDa}\right)$ solution, and their mixtures. The concentrations are chosen so that each species is below their overlap concentration $\mathrm{C}^{*}$. All the samples are with $0.1 \mathrm{M} \mathrm{NaCl}$. The results are shown in Table 1 and Figure 4 . For $10 \mathrm{~g} / \mathrm{L}$ NaPSS $\left(\mathrm{M}_{\mathrm{w}}=68 \mathrm{KDa}\right)$ in $0.1 \mathrm{M} \mathrm{NaCl}$ solution, there is only one mode with the diffusion coefficient $\mathrm{D}=(5.01 \pm 0.13) \mathrm{E}-7 \mathrm{~cm} / \mathrm{s}$. For two types of macromonomers, $\mathrm{D}=(3.88 \pm 0.06) \mathrm{E}-7 \mathrm{~cm}^{2} / \mathrm{s}$ for $5 \mathrm{~g} / \mathrm{L}$ Tetra-PEG-NH $\mathrm{NH}_{2}$ in $0.1 \mathrm{M} \mathrm{NaCl}$ solution and $\mathrm{D}=(3.87 \pm 0.05) \mathrm{E}-7 \mathrm{~cm}^{2} / \mathrm{s}$ for $5 \mathrm{~g} / \mathrm{L}$ Tetra-PEG-NHS in $0.1 \mathrm{M}$ $\mathrm{NaCl}$ solution. When $10 \mathrm{~g} / \mathrm{L}$ NaPSS mixed with $5 \mathrm{~g} / \mathrm{L}$ Tetra-PEG-NHS solution, $\mathrm{D}=(4.05 \pm 0.02) \mathrm{E}-7$ $\mathrm{cm}^{2} / \mathrm{s}$, which is between D of NaPSS alone and D of Tetra-PEG-NHS alone. However, when 10g/L NaPSS mixed with $5 \mathrm{~g} / \mathrm{L}$ Tetra-PEG-NH $\mathrm{N}_{2}$ solution, $\mathrm{D}=(2.71 \pm 0.03) \mathrm{E}-7 \mathrm{~cm}^{2} / \mathrm{s}$, which is lower than both $\mathrm{D}$ of NaPSS alone and D of Tetra-PEG-NH alone, indicating there are bigger aggregates with lower diffusion coefficient show up due to weak complexation between NaPSS and Tetra-PEG-NH . This is the evidence of the weak complexation between the NaPSS and the Tetra-PEG-NH2. When Tetra-PEG-NH $\mathrm{N}_{2}$ macromonomers become the crosslinked gel, the complexation between the $\mathrm{NaPSS}$ and the gel made of Tetra-PEG-NH $\mathrm{N}_{2}$ cannot form aggregates because now Tetra-PEG-NH species is static and frozen in the gel network and cannot freely move. Therefore, the weak complexation between NaPSS and the gel made of Tetra-PEG-NH $\mathrm{N}_{2}$ can only lead to strong fluctuations of the scattered light intensity.

Table 1. Summary of diffusion coefficient $D$ of NaPSS $\left(M_{w}=68 K D a\right)$ solution, macromonomer solutions, and the mixture of NaPSS and macromonomer solutions. All the samples are with $0.1 \mathrm{M}$ $\mathrm{NaCl}$.

\begin{tabular}{|l|l|}
\hline Sample name & Diffusion coefficient D \\
\hline $10 \mathrm{~g} / \mathrm{L}$ NaPSS & $(5.01 \pm 0.13) \mathrm{E}-7 \mathrm{~cm}^{2} / \mathrm{s}$ \\
\hline $5 \mathrm{~g} / \mathrm{L}$ Tetra-PEG-NH & \\
\hline $5 \mathrm{~g} / \mathrm{L}$ Tetra-PEG-NHS & $(3.88 \pm 0.06) \mathrm{E}-7 \mathrm{~cm}^{2} / \mathrm{s}$ \\
\hline $10 \mathrm{~g} / \mathrm{L}$ NaPSS+5g/L Tetra-PEG-NH $\mathrm{N}_{2}$ mixture & $(3.87 \pm 0.05) \mathrm{E}-7 \mathrm{~cm}^{2} / \mathrm{s}$ \\
\hline $10 \mathrm{~g} / \mathrm{L}$ NaPSS+5g/L Tetra-PEG-NHS mixture & $(2.71 \pm 0.03) \mathrm{E}-7 \mathrm{~cm}^{2} / \mathrm{s}$ \\
\hline
\end{tabular}



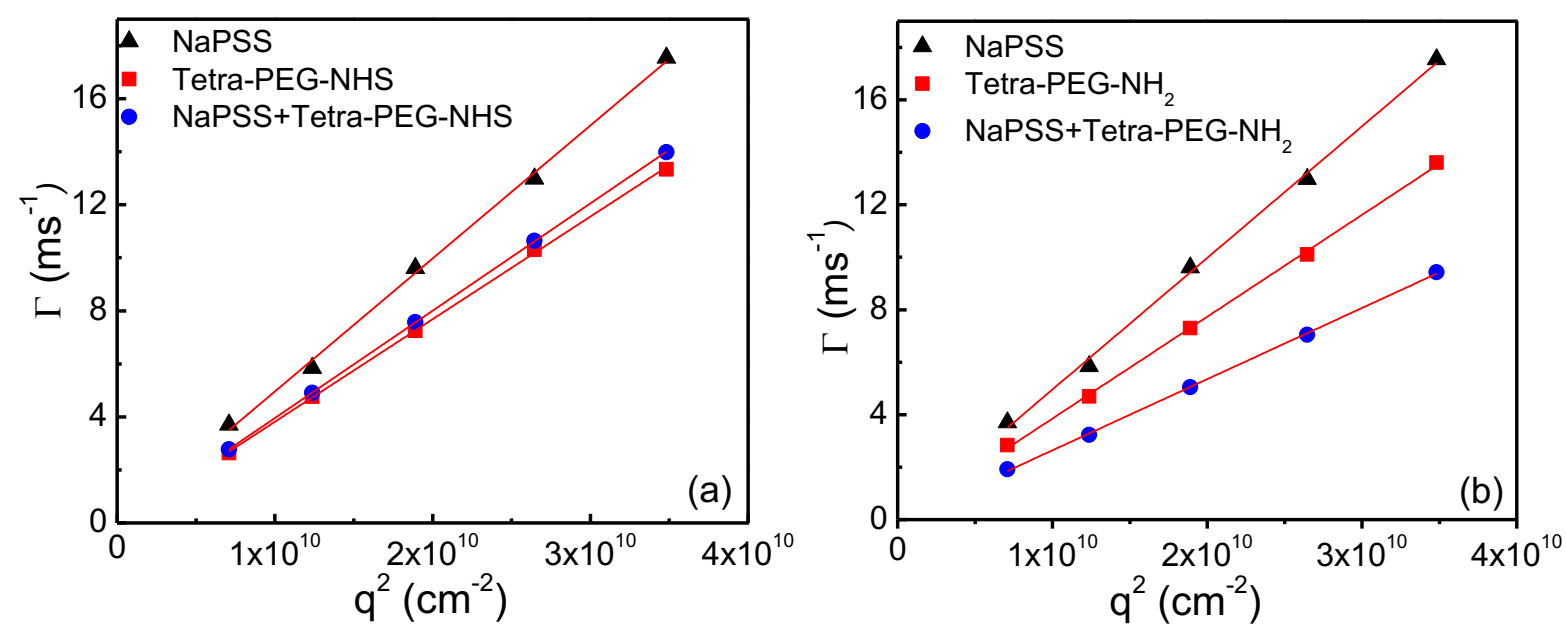

Figure 4. (a) $\mathrm{q}^{2}$ dependence of the relaxation rate $\Gamma$ of $10 \mathrm{mg} / \mathrm{mL} \mathrm{NaPSS}\left(\mathrm{M}_{\mathrm{w}}=68 \mathrm{KDa}\right)$ solution, $5 \mathrm{mg} / \mathrm{mL}$ Tetra-PEG-NHS solution, and the mixture of $10 \mathrm{mg} / \mathrm{mL} \mathrm{NaPSS}$ and $5 \mathrm{mg} / \mathrm{mL}$ Tetra-PEGNHS solution. (b) $q^{2}$ dependence of the relaxation rate $\Gamma$ of $10 \mathrm{mg} / \mathrm{mL} \mathrm{NaPSS}\left(\mathrm{M}_{\mathrm{w}}=68 \mathrm{KDa}\right.$ ) solution, $5 \mathrm{mg} / \mathrm{mL}$ Tetra-PEG-NH $\mathrm{N}_{2}$ solution, and the mixture of $10 \mathrm{mg} / \mathrm{mL} \mathrm{NaPSS}$ and $5 \mathrm{mg} / \mathrm{mL}$ Tetra-PEG-NH solution. All the samples are with $0.1 \mathrm{M} \mathrm{NaCl}$.

\subsection{Dynamics of the guest chains in the neutral gel matrix at intermediate concentration}

Previous results (Figure 2) already show the NaPSS in the Tetra-PEG gel matrix at high concentration $(40 \mathrm{~g} / \mathrm{L})$. Although we cannot obtain the valid scattered light intensity-intensity correlation function $\mathrm{g}_{2}(\mathrm{t})$ measured by DLS at low NaPSS concentration $(5 \mathrm{~g} / \mathrm{L})$ due to the strong fluctuations of the scattered light scattering. But we are still able to get the valid $\mathrm{g}_{2}(\mathrm{t})$ at intermediate NaPSS concentration $(20 \mathrm{~g} / \mathrm{L})$. The correlation function at the scattering angle $30^{\circ}$ and $50^{\circ}$ are shown in figure $5(\mathrm{a})$ and $(\mathrm{b})$ and the best fit of $\mathrm{g}_{1}(\mathrm{t})$ can be accomplished by a sum of one exponential decay, one stretched exponential decay and another exponential decay with much larger decay time (ultra-slow mode), expressed as below:

$$
\mathrm{g}_{1}(\mathrm{t})=\mathrm{a}_{1} \mathrm{e}^{-\Gamma_{1} \mathrm{t}}+\mathrm{a}_{2} \mathrm{e}^{-\left(\Gamma_{2} \mathrm{t}\right)^{\beta}}+\left(1-\mathrm{a}_{1}-\mathrm{a}_{2}\right) \mathrm{e}^{-\Gamma_{3} \mathrm{t}}
$$

where for theg ${ }_{1}(t)$ at scattering angle $30^{\circ}$ (Figure5a), $a_{1}=0.25, \Gamma_{1}=0.23 \mathrm{~ms}^{-1}, a_{2}=0.4, \Gamma_{2}=154.1 \mathrm{~ms}^{-1}$, $\beta=0.36, \Gamma_{3}=550.3 \mathrm{~ms}-1$. And for the $g_{1}(t)$ at scattering angle $50^{\circ}$ (Figure $5 b$ ), $a_{1}=0.31, \Gamma_{1}=0.086 \mathrm{~ms}^{-1}$, $\mathrm{a}_{2}=0.38, \Gamma_{2}=17.92 \mathrm{~ms}^{-1}, \beta=0.37, \Gamma_{3}=115.5 \mathrm{~ms}^{-1}$. The analysis of DLS data on the decay rates at different scattering angles shows that $\Gamma_{1}$ is diffusive with the corresponding diffusion coefficient $D_{1}=(6.26 \pm 0.05) E-7 \mathrm{~cm}^{2} / \mathrm{s}$, which is the gel mode (Figure $5 \mathrm{c}$ ). The second mode is a non-diffusive stretched exponential decay since $\Gamma_{2}$ is not proportional to $q^{2}$ (Figure $5 d$ ). And the value of $\beta$ over all the scattering angles is $\beta=(0.36 \pm 0.03)$ ((Figure $5 f)$. The third non-diffusive mode with a much slower decay rate $\Gamma_{3}$ (ultra-slow mode) comes from the heterogeneity due to the weak complexation between NaPSS and Tetra-PEG gel matrix (Figure 5e). Since the value of $\Gamma_{3}$ is much larger than $\Gamma_{2}$ and $\Gamma_{1}$, so luckily we can decouple the third ultra-slow mode due to heterogeneity from the second stretched exponential mode, which indicates the guest chain dynamics, although 
both the second and third modes are non-diffusive since both $\Gamma_{2}$ and $\Gamma_{3}$ are not proportional to $\mathrm{q}^{2}$.
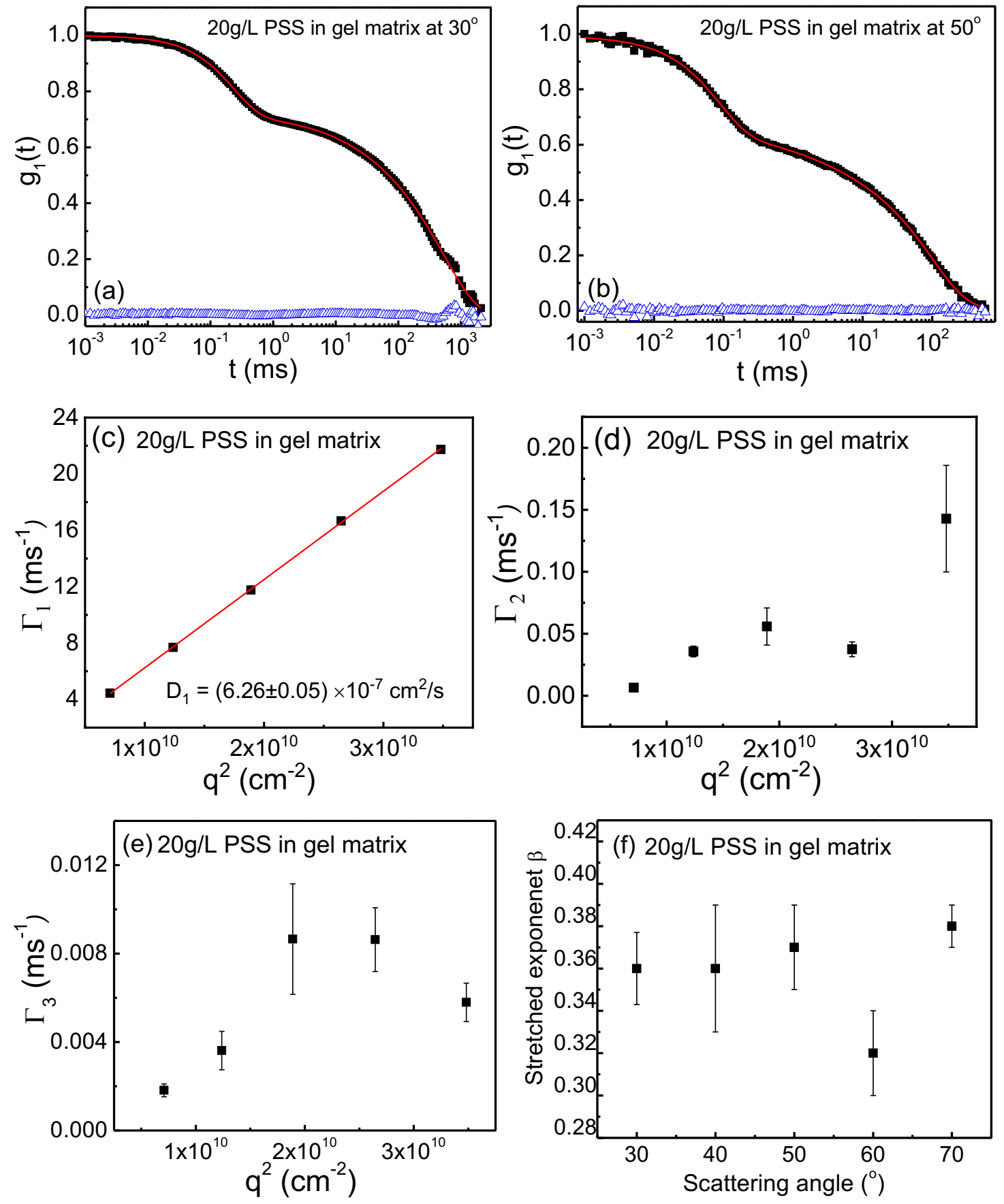

Figure 5. Dynamics of NaPSS chains at $20 \mathrm{~g} / \mathrm{L}$ in the Tetra-PEG gel matrix with $0.1 \mathrm{M} \mathrm{NaCl}$. (a), (b) Normalized field-correlation function $\mathrm{g}_{1}(\mathrm{t})$ at scattering angle $30^{\circ}$ and $50^{\circ}$ respectively. The blue triangles are the residuals between the original data (black) and the fitting curves (red). (c), (d), (e) Fitting results of the relaxation rate $\Gamma_{1}, \Gamma_{2}, \Gamma_{3}$ vs. $q^{2}$ at all angles for the first, second and third mode, respectively. (f) Stretched exponent $\beta$ as a plot of different scattering angles for $20 \mathrm{~g} / \mathrm{L}$ NaPSS in the Tetra-PEG gel matrix with $0.1 \mathrm{M} \mathrm{NaCl}$. 


\section{Conclusions}

As in our previous study using poly(acrylamide-co-acrylate) hydrogels as the host gel, where the mesh size of the gel is not uniform, we find the same phenomenon with similar non-diffusive hierarchical dynamics with the ideal tetra-PEG hydrogels, where the mesh size is uniform. This finding demonstrates that fluctuations in the mesh size of the matrix is not critical to elicit the topologically frustrated dynamical state. Furthermore, since we find the same value of $\beta$ around $1 / 3$ for both systems, we conclude that this new dynamical state is universal and ubiquitous to both charged and uncharged systems.

\section{Acknowledgements}

MM acknowledges financial support from the National Science Foundation (DMR-2004493). DJ acknowledges financial support from Chinese Academy of Sciences.

\section{References}

(1) Sakai, T.; Matsunaga, T.; Yamamoto, Y.; Ito, C.; Yoshida, R.; Suzuki, S.; Sasaki, N.; Shibayama, M.; Chung, U.-i. Macromolecules 2008, 41, 5379.

(2) Matsunaga, T.; Sakai, T.; Akagi, Y.; Chung, U.-i.; Shibayama, M. Macromolecules 2009, 42, 6245.

(3) Kurakazu, M.; Katashima, T.; Chijiishi, M.; Nishi, K.; Akagi, Y.; Matsunaga, T.; Shibayama, M.; Chung, U.-i.; Sakai, T. Macromolecules 2010, 43, 3935.

(4) Shibayama, M.; Li, X.; Sakai, T. Colloid and Polymer Science 2019, 297, 1.

(5) Jia, D.; Muthukumar, M.; Cheng, H.; Han, C. C.; Hammouda, B. Macromolecules 2017, 50, 7291.

(6) Jia, D.; Zuo, T.; Rogers, S.; Cheng, H.; Hammouda, B.; Han, C. C. Macromolecules 2016, 49, 5152.

(7) Han, C. C.; Akcasu, A. Z. Scattering and Dynamics of Polymers: Seeking Order in Disordered Systems; Wiley, 2011.

(8) Provencher, S. W. Computer Physics Communications 1982, 27, 229.

(9) Berne, B. J.; Pecora, R. Dynamic Light Scattering: With Applications to Chemistry, Biology, and Physics; Dover Publications, 2000.

(10) Jia, D.; Muthukumar, M. Journal of the American Chemical Society 2019, 141, 5886.

(11) Jia, D.; Muthukumar, M. Nature Communications 2018, 9, 2248.

(12) Jia, D.; Muthukumar, M. Phys. Rev. Lett. 2021, 126, 057802.

(13) Jia, D.; Muthukumar, M. Macromolecules 2020, 53, 90. 
(14) Morozova, S.; Hamilton, P.; Ravi, N.; Muthukumar, M. Macromolecules 2016, 49, 4619.

(15) Morozova, S.; Muthukumar, M. Macromolecules 2017, 50, 2456.

(16) Jia, D.; Muthukumar, M. Gels 2021, 7.

(17) Muthukumar, M. Journal of Polymer Science Part B: Polymer Physics 2019, 57, 1263.

(18) Gruber, E. Berichte der Bunsengesellschaft für physikalische Chemie 1978, 82, 1019.

(19) McCoy, J. L.; Muthukumar, M. Journal of Polymer Science Part B: Polymer Physics 2010, 48, 2193.

(20) Tanaka, T.; Nishio, I.; Sun, S.-T.; Ueno-Nishio, S. Science 1982, 218, 467.

(21) Berne, B. J.; Pecora, R. Dynamic Light Scattering: With Applications to Chemistry, Biology, and Physics; Dover Publications, 1976.

(22) Schmitz, K. S. An introduction to dynamic light scattering of macromolecules; Academic Press Inc: United States, 1990.

(23) Chu, B. Laser Light Scattering: Basic Principles and Practice; Acad. Press, 1991. 